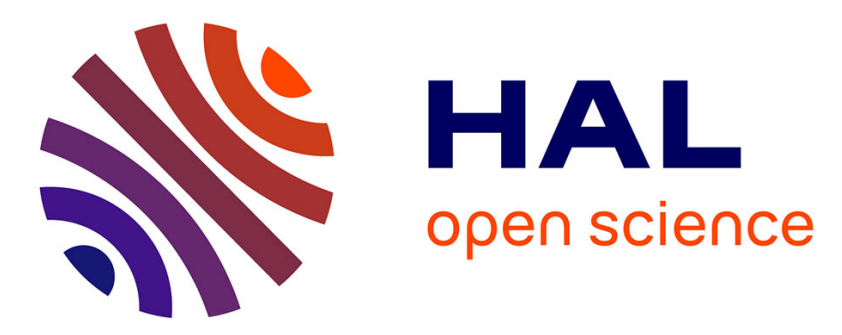

\title{
Conscience de Soi, maintien du Soi et identité humaine au cours de la maladie d'Alzheimer
}

\author{
R. Gil, M.-N. Fargeau, N. Jaafari
}

\section{To cite this version:}

R. Gil, M.-N. Fargeau, N. Jaafari. Conscience de Soi, maintien du Soi et identité humaine au cours de la maladie d'Alzheimer. Annales Médico-Psychologiques, Revue Psychiatrique, 2011, 169 (7), pp.416. 10.1016/j.amp.2011.06.004 . hal-00789298

\section{HAL Id: hal-00789298 \\ https://hal.science/hal-00789298}

Submitted on 18 Feb 2013

HAL is a multi-disciplinary open access archive for the deposit and dissemination of scientific research documents, whether they are published or not. The documents may come from teaching and research institutions in France or abroad, or from public or private research centers.
L'archive ouverte pluridisciplinaire HAL, est destinée au dépôt et à la diffusion de documents scientifiques de niveau recherche, publiés ou non, émanant des établissements d'enseignement et de recherche français ou étrangers, des laboratoires publics ou privés. 


\section{Accepted Manuscript}

Title: Conscience de Soi, maintien du Soi et identité humaine au cours de la maladie d'Alzheimer

Authors: R. Gil, M.-N. Fargeau, N. Jaafari

PII: $\quad$ S0003-4487(11)00163-6

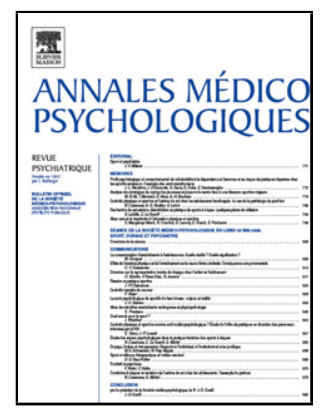

DOI: $\quad$ doi:10.1016/j.amp.2011.06.004

Reference: $\quad$ AMEPSY 1345

To appear in: $\quad$ Annales Médico-Psychologiques

Please cite this article as: Gil R, Fargeau M-N, Jaafari N, Conscience de Soi, maintien du Soi et identité humaine au cours de la maladie d'Alzheimer, Annales mediopsychologiques (2010), doi:10.1016/j.amp.2011.06.004

This is a PDF file of an unedited manuscript that has been accepted for publication. As a service to our customers we are providing this early version of the manuscript. The manuscript will undergo copyediting, typesetting, and review of the resulting proof before it is published in its final form. Please note that during the production process errors may be discovered which could affect the content, and all legal disclaimers that apply to the journal pertain. 
Communication

\title{
Conscience de Soi, maintien du Soi et identité humaine
} au cours de la maladie d'Alzheimer

Self-consciousness, the Self and human identity in Alzheimer's disease R. Gil ${ }^{\text {a, }}$, M.-N. Fargeau ${ }^{\text {a }}$, N. Jaafari ${ }^{\text {b }}$

${ }^{a}$ Unité de Neuropsychologie et de Rééducation du Langage, service de neurologie,

Centre Mémoire de Ressources et de Recherche, CHU de Poitiers, Université de Poitiers, France

${ }^{b}$ Unité de recherche clinique intersectorielle du centre hospitalier Henri Laborit, INSERM CIC 802, CHU et Université de médecine de Poitiers, France

Auteur correspondant : Pr Roger Gil, service de neurologie, unité de neuropsychologie et de rééducation du langage, CHU de Poitiers, 2, rue de la Milétrie, 86021 Poitiers Cedex, France Adresse email : r.gil@chu-poitiers.fr

\section{Résumé}

L’identité est conscience de Soi et s'exprime aussi dans un «être-au-monde » qui manifeste aux autres le Soi. L’évaluation dans une population Alzheimer des trois facettes du Soi (matérielle, sociale, spirituelle) a montré que le Soi social était le plus fragile, et que la sévérité de l'atteinte du Soi était liée à l'apathie et au déficit de la mémoire autobiographique sémantique. Il apparaît aussi que l’ipséité est électivement atteinte par la maladie.

Mots clés : Conscience de soi ; Démence d’Alzheimer ; Identité ; Ipséité

\begin{abstract}
Identity is a part of self-consciousness, which is also expressed as "being in the world" which one in turn shows to others as the Self. The assessment of the Self in a population of patients with Alzheimer's disease, according to a multidimentional definition (physical, social, spiritual), showed that the social self was impaired, and the severity of impairment of the self was correlated to apathy and lack of semantic autobiographical memory. It also appears that ipseity is selectively affected by the disease.
\end{abstract}

Keywords: Alzheimer’s disease; Identity; Ipseity; Self-consciousness 
L’identité humaine est l'ensemble des données historiques, comportementales, cognitives, émotionnelles et sociales qui fondent la singularité de chaque être humain et configurent ainsi son «Soi » ou «Self». Elle est conscience, c’est-à-dire connaissance de ce Soi, mais elle s’exprime aussi dans un « être-au-monde » qui manifeste aux autres le « Soi » ou « Self».

Comprendre comment le cerveau humain organise, exprime et assume le Soi nécessite d'examiner la manière dont le Soi peut être détérioré par les lésions cérébrales. De telles études tentent de comprendre comment les symptômes qui expriment la souffrance cérébrale altèrent la capacité du sujet à maintenir sa cartographie identitaire au sein du monde ainsi que la conscience qu'il a de lui-même [18].

Les démences, par leur fréquence, par la topographie des lésions, par leur évolution progressive peuvent constituer un terrain privilégié d'étude du Soi et de la Conscience de Soi. Certes, toute conscience est, comme l'énonçait Husserl [14], conscience de " quelque chose ». Il s'agit donc d'une conscience perceptive. Mais comme le soulignait déjà Locke [19], cette conscience perceptive est inséparable de la conscience de percevoir. La conscience de Soi rassemble de multiples facettes : conscience de ses perceptions et de ses actions, connaissance du corps, évaluation et estime de Soi, conscience de ses projets (mémoire prospective), conscience morale [10,17]. Tel est pour Locke [19] le socle sur lequel se bâtit l'identité personnelle, le Soi, qui procède d'abord de la prise de conscience qu'a ainsi chaque personne de sa singularité par rapport au monde pensant et agissant qui l'entoure. Mais en même temps, ce sentiment identitaire, cette connaissance de Soi ne sont possibles que parce que la conscience peut, grâce à la mémoire, se reporter vers le passé pour y reconnaître ses pensées et ses actions comme siennes et comme passées. «C’est le même Soi maintenant qu’il était alors » [19]. Et c'est ainsi que la conscience de Soi se hisse dans une Représentation de Soi, un Soi historique, qui peut d'ailleurs se raconter dans un « récit» [24]. On conçoit alors le rôle fondamental joué par la mémoire, et tout particulièrement la mémoire autobiographique dans la construction et la permanence du Soi [6,22]. Hume [13] avait pressenti que la mémoire autobiographique ne pouvait être réduite à une succession d’événements, mais qu’il fallait en outre que puisse se tisser entre eux une continuité fondée sur « des relations de ressemblance, de contiguïté, de causalité » : car l’identité implique la continuité dans le temps donc l'interconnection d'états de conscience se succédant tout au long de la vie. Mais cette mémoire autobiographique n'est pas une mémoire exhaustive qui compilerait un à un tous les 
événements, les actions et les connaissances de la vie. S’il en était ainsi, la mémoire encombrerait tout le champ de notre conscience. C'est ce qu'avait si bien pressenti Jorge Luis Borges [1], en décrivant dans Fictions, l'histoire insolite de ce personnage nommé Funes, qui après avoir été renversé par un cheval fut la victime d'une «mémoire implacable ». Il dit à son interlocuteur: "J’ai à moi seul plus de souvenirs que n’en peuvent avoir eu tous les hommes depuis que le monde est monde. » Je soupçonne, écrit Borges, « qu’il n’était pas très capable de penser ». L’oubli est nécessaire à la mémoire qui se constitue, mais il sera ensuite néfaste à la mémoire constituée. L’identité s’exprime ainsi dans ce que Paul Ricœur [24] avait appelé un récit qui dit et construit «l'identité narrative ». Mais Ricœur [24] ne pense pas comme Locke [19] que la mémoire suffise à surmonter la diversité, c'est-à-dire les changements dans le temps qui affectent tout individu, et à rendre compte à elle seule de la cohérence identitaire dans sa plénitude. Et c’est pourquoi il distingue l’identité-mêmeté de l’identité-ipséité. L’identité-mêmeté est de l’ordre de «l’idem », ou encore de l’ordre de «l'avoir ». Ainsi, en est-il des modifications morphologiques et physiologiques survenant tout au long de la vie, de la jeunesse au vieillissement, et qui n’empêchent pas de concevoir qu'il s’agit d'un même individu. L’identité n'est pas l'identique [24]. L’identité-mêmeté permet la permanence dans le temps, fondée sur l'intégration des changements comme sur leur « continuité ininterrompue ». Elle est de l'ordre du «Que suis-je ? ». Tout autre est l'identité-ipséité qui est de l'ordre de l'être et qui renvoie au maintien de Soi malgré les changements, il en est ainsi de la fidélité à la parole donnée, de la constance dans l'amitié, en somme, dit Ricœur [24], d'une identité éthique que l'on peut prendre dans le sens de comportementale qui rend le sujet capable et comptable de ses actes.

\section{Conscience de Soi et maladie d'Alzheimer}

La maladie d’Alzheimer atteint la mémoire, et en particulier la mémoire épisodique et la mémoire autobiographique ; elle atteint aussi les interactions entre Soi et le Monde car elle atteint le langage, les gestes, les capacités d’interprétation des informations sensorielles. Une étude des diverses facettes de la conscience de Soi dans une population de malades Alzheimer [10] avait ainsi pu montrer que s'il y avait bien une atteinte de la Conscience de Soi, cette atteinte n'était pas globale mais composite. Ainsi, les connaissances sémantiques sur leur identité, leur conscience du schéma corporel et même la conscience de leur état affectif étaient moins souvent atteintes que la conscience de leur maladie, leur mémoire du futur (donc leur capacité à faire des projets) et les jugements moraux. 


\section{Le Self comme « être-au-monde »}

Le Self est certes connaissance de Soi, sentiment de Soi, mais le Self se manifeste aussi par un « être-au-monde » qui fait qu'un individu, par son comportement, ses préférences vestimentaires, ses opinions, exprime un profil identitaire reconnu par autrui. Ces changements du Self comportemental peuvent survenir chez des patients cérébrolésés, et cela apparaît quand l'entourage déclare de la personne malade qu'il « ne la reconnaît pas ». C’est aussi ce qu'exprimaient les collègues de travail de Phineas Gage, qui, revenu parmi eux après l'accident dramatique qui avait gravement lésé son lobe frontal gauche, avait un comportement si modifié qu'ils disaient que «Gage n’est plus Gage » [4,5]. William James [15] distingue trois éléments du Self : Le Soi matériel, le Soi social, le Soi spirituel. Le corps est l'élément central du Self matériel, viennent ensuite les vêtements. Le Self social désigne la manière dont le sujet se comporte à l'égard des groupes sociaux qu'il côtoie car le comportement d'un sujet peut varier selon qu'il est dans sa famille, sur son lien de travail, ou encore en vacances. Le Moi spirituel s'exprime par les opinions morales, politiques, philosophiques, et plus généralement «les tendances qui ont pour objet» le « perfectionnement psychique, intellectuel, moral, ou enfin spirituel ».

Ainsi, repérer une atteinte du Self revient à rechercher par l'interrogatoire de l'entourage si un sujet a " changé », s’il n'est plus reconnu, dans son comportement antérieur au niveau de sa manière de se présenter aux autres, de son comportement à l'égard d'autrui, de ses opinions morales, politiques, religieuses, culturelles. C’est ainsi qu'une étude [8] a porté sur l'évaluation des trois éléments du Self dans une population de malades Alzheimer. Un questionnaire (disponible sur demande) a été soumis à l'aidant principal de chaque malade. Quarante-sept sujets atteints d'une maladie d'Alzheimer d'intensité légère à modérée ont été soumis à un bilan neuropsychologique et comportemental évaluant l'état cognitif (MMS ; Échelle Clinique de Démence - CDR - [12] ; le score frontal - Wisconsin Card Sorting - [21] ; l’apathie - Échelle de Marin - [20] ; la dépression - Échelle de Goldberg [11] ; la mémoire autobiographique - fluence lexicale et fluence événementielle - [23]). La majorité des patients présentaient une altération d'au moins une des trois facettes du Self (43 sur 47). Quand il y avait une, deux ou trois atteintes du Self, le Self social était toujours atteint, et de manière isolée chez un quart des sujets. Un malade sur cinq avait une atteinte des trois facettes du Self. En comparant les malades n'ayant qu'une atteinte du Self social et les malades ayant une atteinte des trois facettes du Self, on pouvait constater par l'analyse 
multivariée que les malades ayant une atteinte des trois facettes du Self avaient une ancienneté plus importante de la maladie, une apathie plus marquée, un déficit plus marqué de la mémoire autobiographique sémantique. Ni le score au Mini Mental State ni le score à l’Échelle clinique de démence de Berg ne différaient significativement entre les deux groupes. Les scores obtenus au questionnaire montraient, après une régression linaire multiple, qu'après ajustement, les variables explicatives de l'atteinte du Self étaient la composante sémantique de la mémoire autobiographique et de l'apathie.

Ainsi, le Soi social est le plus fragile : on sait que l'altération du fonctionnement social et occupationnel fait partie des critères diagnostiques des démences, elle dépend du niveau prémorbide d’activité sociale.

L’altération du Soi matériel et du Soi social est d'autant plus fréquente que la maladie évolue depuis plus longtemps, mais elle n’est pas liée significativement au score au MMSE qui ne donne qu'une évaluation incomplète du fonctionnement cognitif.

L’altération de la mémoire épisodique ne peut expliquer à elle seule la sévérité de l'atteinte du Self, qu'elle soit explorée par l'apprentissage d'une liste de mots ou par l'exploration de la composante épisodique de mémoire autobiographique. Mais la mémoire autobiographique est aussi un savoir qui structure la construction identitaire. Les souvenirs anciens, longtemps mieux préservés dans la maladie d'Alzheimer que les souvenirs récents, sont préférentiellement sémantiques. Ils permettent le maintien du Soi tant qu’ils ne sont pas atteints, ce que suggère le lien entre la sévérité de l’atteinte du Soi, la durée de la maladie et la fluence autobiographique sémantique.

L’apathie est une variable explicative de la sévérité de l’atteinte du Self, mais non la dépression. La littérature indique une fréquence de $25 \%$ à $50 \%$ des malades [17], et ce trouble s'accompagne d'une détresse de l'aidant principal.

\section{Self, identité et vieillissement normal ou sur le mode alzheimérien}

Ainsi, la conscience de Soi envisagée en tant que Self (Myself au sens de William James), constituée d'un ensemble de représentations que l'individu a de lui-même, formée à partir d'expériences vécues et de pensées [16], met la mémoire au centre d’un « Self memory system » [2,3], lié à la mémoire autobiographique. La composante épisodique de la mémoire autobiographique s’appauvrit au cours du vieillissement alors que la composante sémantique est préservée [6]. Ainsi, les sujets âgés «savent » plus qu’ils ne « se souviennent » : or, ces déficits, que l'on pourrait aussi considérer comme une reconfiguration de la mémoire 
épisodique avec l'âge, n’altèrent pas le sentiment d’identité des personnes âgées, et notamment la consistance des représentations de Soi, l'estime de Soi.

Ces constatations rejoignent les analyses des effets du vieillissement analysés selon le modèle proposé par Ricœur [24] et fondé sur la dualité mêmeté/ipséité. Le vieillissement normal impose au sujet d'intégrer ses changements successifs, morphologiques, physiologiques, intellectuels, mnésiques, dans cette continuité existentielle qui permet à l'être humain tout au long de sa vie de se sentir le même alors qu'il n’est jamais «identique » à ce qu’il a été [9]. Il s’agit là de l’identité-mêmeté qui dit la continuité identitaire en dépit des changements. Et témoigne de cette identité-mêmeté et de la connexion entre événements qu'elle suppose, le récit d’une vie, conçu comme « une mise en intrigue » intégrant la permanence dans le temps et permettant ainsi le déploiement d'une « identité narrative». C’est ainsi que s'intègre aussi au cœur de l'identité-mêmeté le caractère, terme désignant dans le vocabulaire de Ricœur [24] «l'ensemble des marques distinctives qui permettent de réidentifier un individu humain comme étant le même», ou encore «l'ensemble des dispositions durables auxquelles on reconnaît une personne ». Ce terme mêle donc ce que l'on a coutume de désigner par les traits de caractère, donc de personnalité, mais aussi les dispositions acquises par l'habitude. C'est pourquoi le caractère n'est pas strictement immuable, mais il assure la permanence dans le temps qui définit la mêmeté. On reconnaît quelqu'un à son caractère et par ce mouvement-là, il touche à l'ipséité en étant en quelque sorte le «Quoi ? » du « Qui ? ».

Ainsi conçu, l'enjeu identitaire du vieillissement «normal » tient à la passivité ou à la souffrance d'un sujet qui, par baisse de l'estime de soi, devient incapable d'accepter et d'assumer les changements anatomiques, physiologiques, intellectuels, affectifs et sociaux liés au vieillissement. C’est alors que l'on glisse du vieillissement normal à la dépression du vieillissement que l’on peut ainsi concevoir comme une mêmeté non acceptée, non assumée, sans rupture de l’ipséité. En effet, les modifications du caractère générées par la dépression n’affectent pas le maintien de Soi («Qui suis-je ? »), mais la Souffrance narcissique d’un Soi désemparé par les changements d’une mêmeté dont le sentiment de continuité et de permanence dans le temps devient souffrance (« Que suis-je devenu ? »).

La maladie d'Alzheimer, cause la plus fréquente, devenue modèle du vieillissement cérébral pathologique, comporte des troubles précoces de la mémoire autobiographique entraînant une fragmentation de la continuité identitaire et un affaiblissement du sentiment d’identité en rapport avec une atteinte élective des souvenirs épisodiques, alors que la plus 
grande résistance de la mémoire sémantique [7] permet aux sujets de conserver des connaissances générales sur eux-mêmes, même s’ils ne peuvent pas se rappeler de souvenirs vécus [22]. L’étude des diverses facettes de la conscience de Soi [9] a ainsi montré la robustesse des connaissances générales sur l’identité (nom, prénom, métier exercé, prénom de l'époux ou de l'épouse...). Ces repères, qui constituent l'ultime rempart identitaire, concernent ainsi les éléments les plus sémantisés de la mémoire autobiographique puisqu’ils intéressent non pas des événements mais des connaissances du sujet sur ce qui a constitué l'histoire de sa vie. Ainsi, le Self tel qu'il se donne à être identifié par Autrui sous ses aspects de Soi matériel, social, spirituel, est d'autant plus sévèrement atteint que la mémoire autobiographique sémantique est altérée [8]. Mais en atteignant déjà la mémoire épisodique, la maladie d’Alzheimer rompt la continuité et la connexion des expériences vécues. Et cela ne peut manquer de retentir sur le maintien de Soi, que Ricœur [24] trouve électivement illustré par la fidélité à la parole donnée. Car ce maintien de Soi chez le sujet normal relève bien d'une identité distincte de la mêmeté et " polairement opposé » au caractère et relève d'une posture qui ne peut tenir compte d'aucun changement, qu'il s’agisse des désirs, des opinions des inclinations. Or, si la mémoire n’en est pas la condition suffisante, comme l'avait relevé Paul Ricœur [24], elle en est néanmoins la condition nécessaire, car la parole donnée prend racine dans une expérience vécue. Ainsi, c’est bien l’identité-ipséité qui est altérée par la maladie d'Alzheimer. L'identité humaine se construit aussi à travers un savoir dire et un savoir-faire ; les troubles du langage font employer au sujet un mot pour un autre selon une dynamique d'appauvrissement lexical de «bas en haut» (bottom-up), qui fait remplacer les mots recherchés par des superordonnés (animal pour chien), tandis que les mots perdent leurs représentations sémantiques pour devenir des coquilles vidées de leur sens : c’est, en sus des troubles de la mémoire, une incapacité d'un autre ordre à mettre en œuvre l’identité narrative. L'apraxie empêche aussi le sujet de témoigner de lui-même à travers son activité gestuelle qui caractérise l'homo faber à travers ce qu'il sait faire avec ses mains. L'identité se bâtit aussi des interactions avec le monde qui entre en nous par les organes des sens pour être interprété c'est-à-dire vu, entendu, touché, senti. L’agnosie spatiale et visuelle va empêcher le sujet de reconnaître le monde qui l'entoure, les repères qui permettent de s'orienter chez soi, dans son quartier, sur un plan, comme elle va rendre le sujet incapable de reconnaître les objets, les paysages, les animaux et enfin les visages, ceux des autres et son propre visage. Et c'est aussi dans ce sillage que surgissent les délires d'identité, et en particulier le syndrome de Capgras. Le déficit de la connaissance de Soi interagit avec le déficit de la reconnaissance de l'Autre et du monde. Ainsi, au fur et à mesure que la maladie progresse, le «maintien de Soi » est mis à 
rude épreuve, ce qui montre l’écart entre la perception du « Qui suis-je ? » et les réponses aux questions explorant sommairement quelques connaissances générales sur l’identité. La déstructuration de l'identité-ipséité est donc au cœur de la problématique du statut éthique du malade Alzheimer dans sa famille et dans la cité. Car le danger serait sans doute de ne voir du sujet que sa détérioration identitaire comptée en débit en se limitant à une éthique palliative additionnant tuteur et personne de confiance. Mais au-delà s'impose le difficile chemin d'une éthique performative qui impose alors de quêter les manifestations identitaires restantes et qui cherche à prolonger l'identité de l'Autre.

\section{Conflit d'intérêt : aucun}

\section{Références}

[1] Borges J.L. Fictions. Paris: Folio; 1993. p.109-18.

[2] Conway MA, Pleydell-Pearce CW. The construction of autobiographical memories in the Self-memory system. Psychol Re 2000;107:261-88.

[3] Conway SA. Memory and the Self. J Mem Lang 2005;53:594-628.

[4] Damasio AR. L'erreur de Descartes. La raison des émotions (un volume). Paris: Odile Jacob; 1995.

[5] Damasio H, Grabowski T, Frank R, Galaburda AM, Damasio AR. The return of Phineas Gage: clues about the brain from the skull of a famous patient. Science 1994;264:1102-5.

[6] Duval C, Eustache F, Piolino P. Self multidimensionnel, mémoire autobiographique et vieillissement. Psychol Neuropsychiatr Vieil 2007;5:179-92.

[7] Eustache F, Piolino P, Giffard B, Viader F, De La Sayette V, Baron JC, Desgranges B. In the course of time: a PET study of the cerebral substrates of autobiographical amnesia in Alzheimer's disease. Brain 2004;127:1549-60.

[8] Fargeau MN, Jaafari N, Ragot S, Houeto JL, Pluchon C, Gil R. Alzheimer's disease and impairment of the Self. Consciousness and Cognition 2010;19:969-76.

[9] Gil R, Ornon C, Arroyo-Anllo E, Bonnaud V. La maladie d'Amzheimer : délabrement identitaire de la personne humaine. In: Identité(s). Université de Poitiers: Maison des Sciences de l’Homme et de la Société, CNRS; 2002. p.41-9.

[10] Gil R, Aroyo-Anllo EM, Ingrand P, Gil M, Neau JP, Ornon C, Bonnaud V. Selfconsciousness and Alzheimer’s disease. Acta Neurol Scand 2001;104:296-300.

[11] Goldberg D, Bridges K, Duncan-Jones P, Grayson D. Detecting anxiety and depression in general medical settings. BMJ 1988;297:897-99. 
[12] Hughes CP, Berg L, Danziger WL, Coben LA, Martin RL. A new clinical scale for the staging of dementia. Br J Psychiatry 1982;140:566-72.

[13] Hume D. A treatise of human nature, edited by DF and MJ Norton. Oxford, New-York: Oxford University Press; 2001.

[14] Husserl E. Méditations Cartésiennes, traduction de Pfeiffer G et Levinas E. Paris: Vrin; 1969.

[15] James W. Précis de Psychologie (un volume). Paris: Marcel Rivière et Cie éditeurs; 1912.

[16] Kihlstrom JF, Cantor N, Albright JS, Chew BR, Klein SB, Niedenthal PM. Information processing and the study of the Self. Adv Exp Soc Psychol 1988;21:145-78.

[17] Landes AM, Spery SD, Strauss ME, Goldmacher DS. Apathy in Alzheimer's disease. J of Am Geriatrics Society 2001;49:1700-7.

[18] Lechevalier B. Polysémie de la conscience. In: Lechevalier B, Eustache F, Viader F (ed). La conscience et ses troubles. Paris: De Boeck Université; 1998. p.9-21.

[19] Locke J. An Essay Concerning Human Understanding (1690), http://oregonstate.edu/instruct/phl302/texts/locke/locke1/Essay_contents.html. Amherst, NewYork: Prometheus Books; 1995.

[20] Marin R, Biedrzycki R, Firinciogullarti S. Reliability and validity of the Apathy Evaluation scale. Psychiatry Research 1991;38:143-62.

[21] Nelson HE. A modified card sorting test sensitive to frontal lobe defects. Cortex 1976;12:313-24.

[22] Piolino P. À la recherche du Self : théorie et pratique de la mémoire autobiographique dans la maladie d'Alzheimer. L'Encéphale 2007;33:33-44.

[23] Piolino P, Desgranges B, Belliard S, Matuszewski V, Lalevée C, De la Sayette V. Autobiographical memory and autonoetic consciousness: Triple dissociation in neurodegenerative diseases. Brain 2003;126:2203-19.

[24] Ricoeur P. Soi-même comme un autre (Oneself as another). Paris: Seuil « Points essais »; 1990.

\section{Discussion}

Pr Houillon - Je tenais tout particulièrement à remercier le Pr Gil pour la façon surtout dont il vient de définir les contours de la maladie d'Alzheimer et d'en évoquer le contenu. Il est en effet nécessaire aujourd'hui de dénoncer les abus de diagnostic concernant cette maladie et qui entraînent à eux seuls des comportements inadaptés, des erreurs d’appréciation, 
des liaisons dangereuses qui associent bien trop rapidement Alzheimer et troubles de mémoire relevant de causes très diverses, et parfois très éloignées d’un diagnostic énoncé avec légèreté, c'est-à-dire sans l'approfondissement clinique qu'il requiert. Sans aller jusqu'à penser à une psychose collective induite par cette polarisation actuelle sur l'Alzheimer, celle-ci ne pourraitelle pas être à l’origine de ces déficits de l’insight dont les conséquences viennent de nous être présentées tout au long de cette matinée?

Pr Allilaire - Je remercie chaleureusement le Pr Gil pour son exposé qui montre l’importance de l’apport des sciences humaines à la réflexion neurologique.

Le propos qu'il a tenu sur l'identité humaine et ses altérations dans l'Alzheimer va donc dans le sens d'une réfutation de la conception anosognosique de l'insight - si je l'ai bien compris.

Est-ce un plaidoyer pour la conception multidisciplinaire de l'insight dont pourrait se servir la psychiatrie pour développer ses travaux sur les altérations de l’insight dans les pathologies psychiatriques?

Réponse du Rapporteur - Le diagnostic de maladie d'Alzheimer se doit d'être rigoureux. C’est nécessaire pour mieux prendre en charge les malades et pour prescrire les médicaments adaptés. Et il est clair que les déficits de l’insight dépendent de la pathologie en cause : le déficit de l’insight observé par exemple dans les démences fronto-temporales n’est pas du même ordre que celui observé dans la maladie d'Alzheimer.

L'anosognosie est une conception trop restrictive de l'insight dont la conception doit bien sûr être multidisciplinaire. 